\title{
Riester-Vorsorge zwischen Theorie und empirischer Evidenz: Wie hilfreich ist das Lebenszyklusmodell?
}

\author{
Im Zuge der Riester-Rente wird in der Öffentlichkeit und den Medien, in Politik und \\ Wissenschaft viel über die Notwendigkeit diskutiert, eine private Altersvorsorge abzu- \\ schließen. Das empirisch beobachtbare Vorsorgeverhalten der heute Erwerbstätigen und \\ künftigen Rentenbezieher ${ }^{\bullet}$ widerspricht jedoch in vielerlei Hinsicht den Erwartungen, \\ die Politiker, aber auch Forscher, hatten und haben. Eine wesentliche Ursache hierfür \\ könnte das vorherrschende Leitbild eines ökonomisch rationalen Verbrauchers sein, der \\ alle Konsumentscheidungen über den Zeitverlauf hinweg umsichtig trifft. Dieses Leitbild \\ wurde in der angelsächsischen Forschung als „Lebenszyklusmodell“ ausformuliert. Dabei, \\ so zeigt dieser Beitrag, eignet sich dieses Modell kaum zur Vorhersage von \\ Altersvorsorgescheidungen. ${ }^{\circ}$
}

FELIX WILKE

\section{Einleitung}

Der Paradigmenwechsel, der sich mit der Einführung der Riester-Rente im Bereich der deutschen Altersvorsorge vollzogen hat (vgl. u.a. Hinrichs 2004), ist in seiner sozialpolitischen Relevanz kaum zu unterschätzen. Denn ob ein lebensstandardsicherndes Einkommen als normatives Gerüst der Altersversorgung weiterhin in weiten Teilen der Gesellschaft erreicht werden kann, hängt nunmehr auch vom Vorsorgeverhalten der Verbraucher ab. Das „alte“ Rentensystem in Deutschland hingegen befand sich bei einem vormals durchschnittlich höherem und qua Umlageverfahren finanzierten Rentenniveau fast ausschließlich in Verantwortung der Sozialversicherung. Der Entscheidungsspielraum der Bürger bei der Gestaltung ihrer Rente war damit sehr begrenzt bzw. auch nur punktuell gefordert. Seit der Rentenreform 2001 obliegt es dagegen aktiven Nutzern, ihre Versorgung im Alter mitzugestalten. ${ }^{3}$ Sie müssen sich in einem noch neuen Altersvorsorgemarkt kompetent bewegen, um sich mit den passenden Produkten für das Alter abzusichern. Diese Aufgabe ist nicht leicht, da in einem Wohlfahrtsmarkt oft diffuse und nicht selten sich widersprechende „multiple Nutzeridentitäten“ auftreten (vgl. Ewert in diesem Heft). Es ist daher notwendig, das Vorsorgeverhalten der einzelnen Nutzer möglichst gut zu verstehen - auch um gesellschaftlich unerwünschte Konsequenzen zu vermeiden. Das Verständnis vom Nutzer war bisher aller- dings sowohl in der politischen Praxis als auch in der sozialwissenschaftlichen Forschung auf einige wenige voraussetzungsvolle Verhaltensannahmen reduziert. Den Kern bildet dabei der rational handelnde, weitsichtige Akteur. In der Forschung wird diese Sicht besonders prominent von der in der ökonomischen Spartheorie vorherrschenden Lebenszyklushypothese (im Folgenden: LZH) vertreten (Legros 2006). Dieser Beitrag zeigt dagegen auf, dass das in Politik und Wirtschaft unterstellte strategisch planende Verhalten sich nicht mit den empirischen Erfahrungen deckt. Viele der Vorhersagen der LZH über das Sparverhalten treffen auf das tatsächliche Vorsorgeverhalten der Deutschen nicht zu. Damit aber wird zweifelhaft, ob die

\footnotetext{
Zur Vereinfachung des Leseflusses wird im Folgenden das männliche Geschlecht genannt. Gemeint sind jeweils beide Geschlechter gleichermaßen.

2 Eine erste Fassung dieses Beitrags wurde auf einem Autorenworkshop für dieses Schwerpunktheft der WSI-Mitteilungen diskutiert. Ich danke den Teilnehmern für die vielen hilfreichen Kommentare. Besonderer Dank gilt Stephan Köppe und Ingo Bode für die konstruktiv-kritischen Anmerkungen zum Manuskript sowie Dirk Schuchardt für seine Hinweise aus dem Praxisalltag eines Beraters.

(3) Siehe zu den Folgen der Reformmaßnahmen für die betriebliche Altersversorgung und ihre Nutzer auch die Beiträge von Blank und Burger in diesem Heft.
} 
politischen und gesellschaftlichen Versorgungsziele im Rahmen des derzeitig eingeschlagenen Weges für breite Bevölkerungsschichten realisiert werden können.

Der Beitrag beginnt mit einer Darstellung der Lebenszyklushypothese, die auf dem Leitbild des ökonomisch rationalen Verbrauchers basiert (Abschnitt 2). Die der LZH zugrunde liegenden theoretischen Annahmen erscheinen zunächst gut geeignet, hypothesenhafte Aussagen zum Verbraucherverhalten im Bereich der Altersvorsorge zu formulieren (3). Im empirischen Teil (4) wird dann allerdings anhand des Planungsprozesses und des Altersvorsorgeverhaltens der aktuell Erwerbstätigen bzw. Erwerbsfähigen und künftigen Rentenbezieher gezeigt, dass die theoretischen Annahmen und Vorhersagen des Modells empirisch kaum zutreffen. Abschließend werden die Ergebnisse im Hinblick auf deren theoretische und praktische Implikationen diskutiert (5).

\section{Die Lebenszyklushypothese}

Den Standardzugang zur Analyse von individuellen Sparentscheidungen bildet in den Wirtschaftswissenschaften die LZH von Modigliani/Brumberg (1954). Die Grundaussage des Modells ist bestechend einfach: Akteure versuchen durch ihre Sparentscheidungen über die Perioden ihres Lebenslaufs hinweg einen konstanten Konsum(nutzen) zu realisieren. Nur unter der Bedingung, dass der momentane Konsum dem Permanteneinkommen entspricht, ist der Nutzen des Individuums über die Zeit maximal. Abbildung 1 stellt das Optimierungskalkül in einem sehr einfa-

ABB. 1

\section{Schema der Lebenszyklusthese}

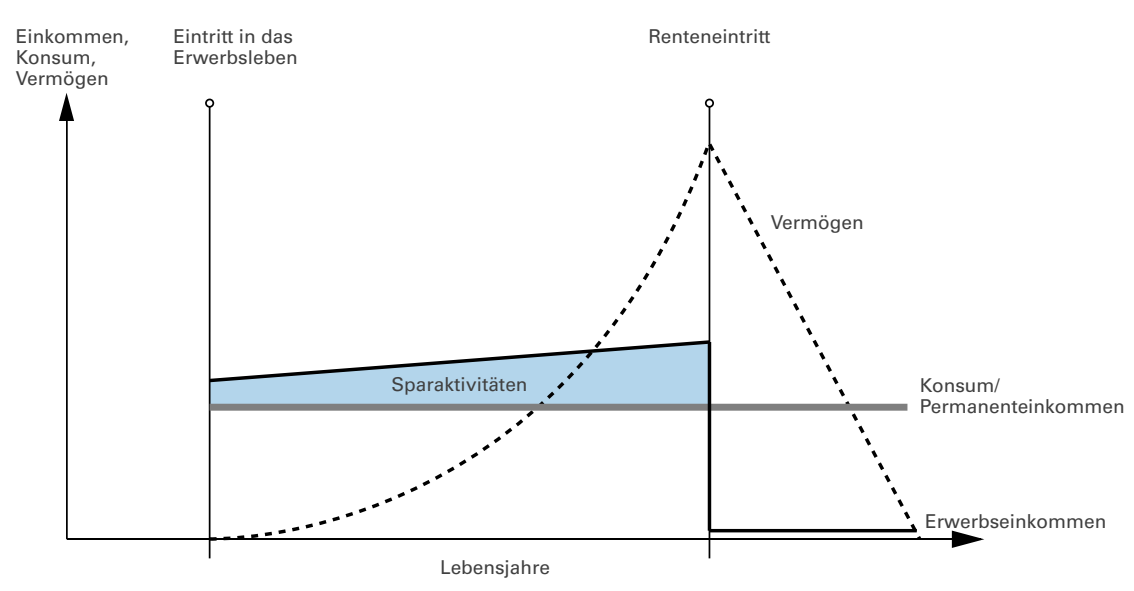

Quelle: Darstellung des Autors. chen Grundmodell unter der zusätzlichen Annahme eines steigenden Erwerbseinkommens schematisch dar. In dieser einfachen Fassung der LZH sind sämtliche Sparaktivitäten (hellblaue Fläche) über den Lebenslauf hinweg auf die Rentenphase ohne Erwerbseinkommen ausgerichtet, damit ein konstanter Konsum(nutzen) auch im Rentenalter aufrechterhalten werden kann (Legros 2006, S. 183ff.). Viele Autoren, die mit Lebenszyklusmodellen arbeiten, sehen daher die Altersphase als die wesentliche Triebkraft für individuelle Sparentscheidungen (allen voran die klassischen Arbeiten von Modigliani/Brumberg 1954 und Feldstein 1974).

Diese einfache Variante der LZH hat mittlerweile zahlreiche Modifikationen erfahren, um realistischere Kontextbedingungen zu integrieren. Allen diesen Änderungen (Vererbungsmotive, Zinsveränderungen, Kreditrestriktionen sowie die Unsicherheit von Entscheidungen; vgl. dazu ausführlich Wilke 2010) ist jedoch gemein, dass sie die Planung der Altersvorsorge kaum beeinflussen sollten. Aus dem Blickwinkel der theoretischen Konzeption des Lebenszyklusmodells sollte sich die Basisvariante deshalb für die Erklärung der Altersvorsorgeersparnisse besonders gut eignen.

Diese theoretisch hoch abstrakte Formulierung der LZH hat sich in der politischen Praxis zu einem wirkmächtigen Konstrukt entwickelt. Auch wenn der explizite Bezug zur LZH eher sporadisch erfolgt, so finden sich die Verhaltensannahmen der LZH in der institutionellen Ausgestaltung der privaten Altersvorsorge doch deutlich wieder. So wird der „Lebenszyklussparer“, der seinen Konsum über den Lebenslauf glättet, seit den Riester-Reformen aktiv gefordert. Die Glättung des Lebenskonsums, die zuvor weitestgehend durch die staatliche Rentenversicherung automatisch erfolgte, muss nun aktiv durch den Nutzer im Rahmen eines „welfare mixes“ (Bode 2007) sichergestellt werden. Um die Absicherung auch weiterhin flächendeckend zu erreichen, wird mittels ökonomischer Anreize (ergo: staatliche Zuschüsse) nachjustiert. Wobei die Konstruktion dieser Anreize auf der Annahme beruht, dass die Akteure ihren Lebenskonsum rational planen. Mit dem Leitbild eines rationalen Konsumenten operieren aber nicht nur politische Akteure, sondern auch Rentenberater oder die jeweiligen Anbieter von zusätzlichen privaten Rentenversicherungen (vgl. Blank 2011, S. 11; Duš/Maurer 2007). Die Versicherungsbranche geht beispielsweise von sehr hohen Rationalitätsannahmen aus, was in der Rentenkalkulation über die Lebenserwartung ihrer Kunden deutlich wird. Versicherungen nehmen an, dass Leute auf ihre Lebenserwartung quasi „wetten“, also in der Regel völlig ungesicherte Wahrscheinlichkeitsannahmen über ihre eigene Lebenserwartung treffen, und damit Rentenversicherungen nur hoch selektiv abgeschlossen werden (Hühne/Balodis 2010, S. 30).

Untersuchungen, ob das theoretische und - wie bereits erwähnt - die institutionelle Ausgestaltung der privaten Altersvorsorge sehr wohl beeinflussende Gerüst der LZH allerdings auch einer empirischen Überprüfung standhält, gibt es noch nicht. In der bisherigen empirischen Forschungsliteratur bildet die LZH zwar häufig den theoretischen Rah- 
men; diese rückt dann aber zugunsten von Zusammenhängen abseits der engen LZH in den Hintergrund (so z. B. Leinert 2003; Börsch-Supan et al. 2006). Als bezeichnend kann die „Behavioral Life Cycle Hypothesis“ gelten (Shefrin/ Thaler 1988). Sie bedient sich der LZH als Idealfall und widmet sich dann Abweichungen, zu denen es aufgrund kognitiver Einschränkungen aus dem Blickwinkel der Verhaltensökonomie kommt. Das Lebenszyklusparadigma wird dabei als nicht mehr weiter zu überprüfender theoretischer Kern belassen. In dieser Forschungsrichtung wurde vor allem versucht zu zeigen, dass es bei Bevölkerungsgruppen mit Informations(verarbeitungs)problemen zu Abweichungen vom „Lebenszyklussparer“ kommt (Nüchter et al. 2008, S. 120ff.; Leinert 2003; Oehler/Werner 2008; Kohlert/Oehler 2009). Über die empirische Erklärungsleistung der LZH für Altersvorsorgeentscheidungen kann diese Forschung höchstens indirekt etwas sagen. Dabei bietet die einfache Grundstruktur der LZH von ihrem Anspruch her durchaus Möglichkeiten, die Situation von Verbrauchern in Beziehung zu ihrem Vorsorgeverhalten zu setzen und daraus Vorhersagen abzuleiten. Darauf geht der folgende Abschnitt ein.

\section{Hypothesen zur Vorsorge von Lebenszyklussparern}

Im Folgenden geht es um die Frage, welches Vorsorgeverhalten in der Bevölkerung zu erwarten wäre, wenn sich die Verbraucher im Mittel ökonomisch rational verhalten würden.

\subsection{Einkommen und Vorsorgeverhalten}

Beginnend mit dem Einkommen gibt es eine klare Beziehung zum Vorsorgeverhalten, die sich aus der LZH ableiten lässt. Jedoch ist im Rahmen der LZH nicht das Einkommen per se entscheidend, sondern die Differenz zwischen aktuellem Erwerbseinkommen und dem eigenen Durchschnittseinkommen (Permanenteinkommen). Sämtliches Einkommen, welches über das permanente Einkommen hinausgeht, wird - so die Annahme der LZH - gespart. Das oft in Querschnittsstudien beobachtete überproportional mit dem Einkommen ansteigende Sparverhalten (vgl. Dynan et al. 2004) wird daher nicht auf generell steigende Sparmöglichkeiten, sondern auf vorübergehend günstige Einkommenspositionen zurückgeführt. Zwei Personen mit identischen aktuellen Einkommen werden im Rahmen der LZH daher gänzlich andere Sparentscheidungen treffen, wenn sie sich bezüglich ihrer Permanenteinkommen unterscheiden.

Das Permanenteinkommen ist allerdings empirisch nur schwer zu erfassen. Bei hohen Sparbeträgen in oberen Einkommensgruppen ist nicht unmittelbar klar, ob es sich um eine vorübergehend günstige Einkommensposition entsprechend der LZH handelt oder nicht (Smyth 1993). Um zu erfassen, ob beispielsweise eine geringe Sparneigung bei Haushalten mit niedrigen Einkommen durch eine vorübergehend negative Einkommensposition hervorgerufen wird, sind im Modell Indikatoren für die Einkommensentwicklung integriert. Haushalte mit positiven Einkommenserwartungen sollten unter Kontrolle des aktuellen Einkommens weit geringere Sparanstrengungen zeigen, da sie von einem höheren Permanenteinkommen ausgehen.

Neben den rein einkommensbezogenen Sparmöglichkeiten verändert die staatliche Förderung die Anreize zum Alterssparen erheblich. So schwankt die effektive Förderung sehr stark mit dem Einkommen. Die fixen staatlichen $\mathrm{Zu}$ schüsse im Rahmen der Riester-Rente wirken vor allem in den unteren Einkommensschichten als starker monetärer Sparanreiz. Dort ist die Rendite aus den Einzahlungen extrem hoch. Für die mittleren Einkommensgruppen sind weniger die fixen Zulagen entscheidend als vielmehr die Möglichkeit, die Altersvorsorgebeiträge steuerlich als Sonderausgabenabzug geltend zu machen (Börsch-Supan et al. 2006, S. 42). Der Sonderausgabenabzug ist allerdings auf $2.100 €$ begrenzt. D. h. für Haushalte mit hohem Einkommen wird die Riester-Vorsorge aufgrund der Deckelung der Steuervorteile wiederum etwas unattraktiver.

Für die Riester-Rente lässt sich aus der Kombination von Einkommens- und Fördereffekten ein hügelförmiger Zusammenhang vermuten. Zunächst steigt die Altersvorsorgeneigung mit dem Einkommen an, und zwar bis zu einem Punkt, ab dem die staatliche Förderung unattraktiver wird. Für hohe Einkommen sollte daher wieder eine geringere Inanspruchnahme staatlich geförderter Altersvorsorge beobachtbar sein, wobei hier eine Verlagerung hin zu anderen Sparformen erfolgen sollte. Bei geringen Einkommen sind zwar die Förderquoten sehr hoch, allerdings sind die Sparmöglichkeiten der Haushalte auch äußerst begrenzt.

\subsection{Alter und Vorsorgeaktivitäten}

Welcher Zusammenhang zwischen dem Alter und Vorsorgeaktivitätenlässt sich aus einem strikten Lebenszyklusmodell ableiten? Grundsätzlich sollte es keine direkte Verbindung zwischen beiden Größen geben (ähnlich Legros 2006). Allerdings lässt sich unter der Annahme, dass mit dem Alter auch das Einkommen steigt (Blossfeld 1989), dennoch ein indirekter Effekt des Alters vermuten, denn das Alter liefert Informationen über die individuelle Einkommensentwicklung. Beispielsweise sollte eine jüngere Person Einkommenssteigerungen erst noch vor sich haben (Abbildung 1) und insofern Sparanstrengungen auf die Zukunft verschieben. Für eine ältere Person mit dem gleichen Einkommen gilt dagegen umgekehrt, dass das momentane Einkommen bereits nah am maximal erreichbaren Einkommen ist und entsprechend viel gespart werden sollte.

Darüber hinaus hat die Rentenreform von 2001 für die verschiedenen Altersgruppen unterschiedliche Auswirkungen. So wird es erst langfristig zu einer deutlichen Absenkung bei der gesetzlichen Rentenversicherung kom- 
men (Oehler 2009, S. 77ff.). Deshalb sind vor allem jüngere Haushalte angesprochen, zusätzliche Alterssparanstrengungen zu unternehmen. Darüber hinaus ist die Rentenreform noch nicht sehr alt und die bisherigen Riester-Laufzeiten sind zwangläufig noch sehr begrenzt. Insbesondere für ältere Kohorten ist die private Altersvorsorge eher unattraktiv. Noch recht generöse gesetzliche Renten und eine kurze Ansparzeit, auf die auch noch die hohen Abschlusskosten umgelegt werden (Oehler 2009, S. 124ff.), lassen den Nutzen einer privaten Altersvorsorge für ältere Beschäftigte aktuell als begrenzt erscheinen.

Werden die altersspezifischen Anreize zusammengefügt, so ist auch für das Alter ein hügelförmiger Effekt zu vermuten. Die zunächst mit dem Alter steigende Neigung, die neu eingeführten staatlichen Vorsorgeprodukte abzuschließen, sollte einige Jahre vor Renteneintritt wieder abnehmen. Der kurvilineare Effekt sollte bei nicht staatlich geförderten Altersvorsorgeprodukten (z. B. private Rentenversicherungen) deutlich geringer sein, da diese Produkte schon lange existieren und deshalb möglicherweise schon im jungen Alter kontrahiert wurden.

\subsection{Weitere Determinanten: Lebenserwartung, Vermögen, Planungshorizont}

Die Altersvorsorgeaufwendungen eines „Lebenszyklussparers" sollten ganz entscheidend von der individuellen Lebenserwartung abhängen, denn das notwendige Vermögen zur Aufrechterhaltung des Konsums im Ruhestand variiert erheblich mit der Anzahl der erwarteten Rentenjahre. Die subjektive Lebenserwartung stellt also im Rahmen der LZH eine Kerngröße des Kapitalbedarfs dar. Empirisch sollte ein rational agierender Akteur daher im Fall einer subjektiv hohen Lebenserwartung, sofern er sich entsprechend der LZH verhält, erheblich mehr für die Altersvorsorge tun als vergleichbare Akteure, die subjektiv ein kürzeres Leben erwarten. Diese Anreizstruktur ändert sich auch durch die bei der Riester-Rente vorgeschriebenen lebenslangen Renten nicht. Personen mit einer hohen Lebenserwartung profitieren während der Auszahlungsphase durch die Umverteilung von den kürzer zu den länger Lebenden.

Neben der subjektiven Lebenserwartung spielt bereits vorhandenes Vermögen ebenfalls eine wichtige Rolle. Entscheidend für das Sparverhalten ist das gesamte Vermögen und nicht nur das altersvorsorgespezifische Vermögen. Mit dieser Einsicht argumentieren beispielsweise Forschungsarbeiten, die sich kritisch mit den Spareffekten von staatlichen Programmen wie der Riester-Rente auseinandersetzen. So gehen Corneo et al. (2009) davon aus, dass durch die Riester-Förderung lediglich das Portfolio umgeschichtet wird, statt mehr zu sparen. Aus der Perspektive der LZH sollte daher eine Substitutionsbeziehung zwischen Altersvorsorgeaufwendungen und anderen Ersparnissen bestehen (Lang 1998, S. 153ff.): Personen, die bereits Vermögen haben, benötigen schlicht weniger Altersvorsorgeprodukte. Das Gleiche gilt für bereits vorhandene Vorsorgeansprüche.
Sowohl hohe gesetzliche Rentenansprüche als auch andere - nicht-staatliche - Alterseinkommensquellen sollten die Sparaktivitäten im Rahmen der Riester-Vorsorge deutlich herabsetzen (z. B. Feldstein 1974).

Zwar im engen Sinne nicht mehr Bestandteil der LZH, aber dennoch in der Logik der Theorie verhaftet, ist der individuelle Zeithorizont. Es ist davon auszugehen, dass Personen mit einem längeren Planungshorizont viel vorausschauender im Hinblick auf Altersvorsorgefragen handeln. Als myopische, also eher auf kurze Sicht operierende Akteure lassen sich dagegen jene einordnen, die Tätigkeiten mit unmittelbaren Resultaten wichtiger einstufen als Tätigkeiten mit zukünftigen Resultaten und deshalb auch weniger Altersvorsorge betreiben (vgl. Frederick et al. 2002; Carroll/Samwick 1997).

Es wurde deutlich, dass die Lebenszyklushypothese (LZH) sich in theoretischer Sicht durchaus eignet, um Vorhersagen über das Verhalten der Verbraucher im noch neuen Altersvorsorgemarkt zu treffen. Die spannende Frage aber ist, ob das hier gewonnene Bild vom rationalen Nutzerverhalten auch der Wirklichkeit entspricht, also eine empirisch zutreffende Beschreibung ist, oder ob nicht andere Nutzerrollen als die des rationalen Verbrauchers das Altersvorsorgeverhalten besser erfassen können. Dies zu klären, ist Gegenstand des nächsten Abschnitts.

\section{Empirische Auswertung}

Die empirische Überprüfung der Hypothesen über den „Lebenszyklussparer" erfolgt in zwei Schritten. Zunächst wird deskriptiv untersucht, in welchem Umfang Altersvorsorge einem Planungsprozess unterliegt (4.1). Dann wird in einem zweiten Schritt gezeigt, inwieweit sich die LZH zur Erklärung des tatsächlichen Vorsorgeverhaltens eignet (4.2).

Als Datengrundlage dient vorwiegend die SAVE-Panelstudie $^{\circledR}$ aus dem Jahr 2008 (Börsch-Supan et al. 2008). Sie beruht auf einer Stichprobe von 2608 repräsentativ ausge-

4 "Die SAVE-Panelstudie erhebt detaillierte, quantitative Informationen, um das Spar- und Altersvorsorgeverhalten deutscher Haushalte besser verstehen zu können. Die SAVE Daten enthalten traditionelle ökonomische Variablen (wie zum Beispiel Einkommen, Spareinlagen und Vermögenshöhe) sowie für das langfristige Planen relevante sozio-psychologische und gesundheitliche Aspekte. Das Panel begann im Jahr 2001 mit methodischen Experimenten und wird seit 2005 jährlich erhoben. 2009 wurden Module zur Finanzkrise und zum finanziellen Allgemeinwissen eingefügt." (http://forschung.deutsche-rentenversicherung.de/FdzPortalWeb/FdzLitDispDetail.do?id=487). Die SAVE-Panelstudie wird durchgeführt vom ehemaligen Mannheimer Forschungsinstitut Ökonomie und Demographischer Wandel (MEA) an der Universität Mannheim. Seit Juli 2011 befindet sich das MEA in München (Munich Center for the Economics of Aging). 


\section{Kenntnisse des erwarteten Rentenniveaus und des Vorsorgebedarfs}

\begin{tabular}{|c|c|c|c|c|c|c|c|c|c|}
\hline \multirow[b]{2}{*}{ Schätzung zum } & \multicolumn{3}{|c|}{ Alle Befragten } & \multicolumn{3}{|c|}{ mit Riester-Vertrag } & \multicolumn{3}{|c|}{$\begin{array}{l}\text { nicht staatlich geförderte } \\
\text { private Altersvorsorge }\end{array}$} \\
\hline & Ja & $\%$ & Gesamt & Ja & $\%$ & Gesamt & $\mathrm{Ja}$ & $\%$ & Gesamt \\
\hline Rentenniveau aus GRV möglich & 832 & 53.7 & 1.548 & 292 & 62.9 & 464 & 141 & 68.1 & 207 \\
\hline Rentenniveau aus GRV und Privat möglich & 490 & 31.7 & 1.548 & 204 & 44.0 & 464 & 109 & 52.7 & 207 \\
\hline bereits privaten Altersvorsorgebedarf ermittelt? (SAVE 09) & 342 & 26.0 & 1.317 & 150 & 35.9 & 418 & 92 & 42.0 & 219 \\
\hline \multicolumn{7}{|c|}{$\begin{array}{l}\text { Anmerkungen: Datenbasis SAVE 2008/2009. } \\
\text { Quelle: Berechnungen des Autors; ohne Selbstständige und Rentner, ungewichtet, nur gültige Werte. }\end{array}$} & \multicolumn{3}{|c|}{ WSI Mitteilungen } \\
\hline
\end{tabular}

wählten Haushalten in Deutschland. Grundlage der Auswertung ist eine Kombination aus Variablen des befragten Haushaltsvorstandes sowie einiger Indikatoren auf Haushaltsebene. Damit werden individuumsbezogene Hypothesen teilweise nur auf der Haushaltsebene überprüft. Zwar kommt es dadurch zur Vermischung verschiedener Betrachtungsebenen, allerdings sind viele monetäre Indikatoren nur auf der Haushaltsebene sinnvoll auszuwerten. Um eine mögliche Verzerrung der Ergebnisse zu vermeiden, wurde die Haushaltsstruktur in den multivariaten Modellen mit berücksichtigt.

\subsection{Deskriptive Analyse}

Wie aus der Darstellung der Theorie deutlich wurde, erfordert ein lebenszyklisches Sparverhalten erheblichen Planungsaufwand im Hinblick auf den Ruhestand; daher sollte bei den Verbrauchern Wissen über die Versorgungslage im Alter und den Vorsorgebedarf vorhanden sein. Wie aus Tabelle 1 deutlich wird, können allerdings lediglich die Hälfte der befragten Personen Schätzungen darüber abgeben, wie hoch ihre gesetzliche Rente ausfallen könnte. Dieser per se schon ernüchternde Befund gewinnt nochmals an Gewicht, wenn man einbezieht, dass einzig Schätzungen - unabhängig von der Richtigkeit der Angaben - von den Befragten erbeten worden sind. Werden die Haushalte zu ihrem kombinierten Alterseinkommensniveau aus gesetzlicher und privater Vorsorge befragt, so kann nur noch weniger als ein Drittel der Haushalte eine Schätzung abgeben. Und noch weniger Haushalte haben tatsächlich den notwendigen Bedarf an privater Vorsorge ermittelt. D.h.: Bei einer beträchtlichen Anzahl der Haushalte fehlt grundsätzliches Wissen, um überhaupt Planungen für die Altersvorsorge durchführen zu können. Unter jenen Befragten, die zusätzliche Altersvorsorge betreiben, ist das Wissen über das eigene Rentenniveau zwar weiter verbreitet, weist aber dennoch erhebliche Lücken auf. So ist auch in dieser Gruppe lediglich die Hälfte der Haushalte in der Lage, eine Schätzung zum Einkommensniveau im Alter abzugeben - noch weniger haben bisher ihren Vorsorgebedarf ermittelt.
Tabelle 1 verdeutlich, dass sowohl die Planung als auch das zur Planung notwendige Wissen nur bei einem Teil der Bevölkerung vorhanden sind und selbst bei jenen Befragten, die zusätzlich privat vorsorgen, nicht vorausgesetzt werden können. Als Kritik an der LZH ist eine solche Beobachtung allerdings noch nicht ausreichend. So ist es durchaus denkbar, dass sich Leute, ohne es direkt zu planen, entsprechend der LZH verhalten (Binswanger/Carman 2009). Dazu wird im nächsten Schritt das Altersvorsorgeverhalten genauer analysiert.

\subsection{Multivariate Analyse}

Im Folgenden werden die in Abschnitt 3 skizzierten Hypothesen in einem multivariaten Modell getestet. Es werden vier Modellvarianten vorgestellt (Tabelle 2). So werden mithilfe zweier logistischer Regressionsmodelle die Chancen für das Vorhandensein einer Riester-Rente (1) sowie das Vorhandensein eines staatlich geförderten oder auch privaten Rentenprodukts (2) modelliert. ${ }^{\ominus}$ Anschließend wird für dieselben Variablen bei der Gruppe der Haushalte, die ein oder mehrere entsprechende Produkte besitzt, untersucht, inwiefern die unabhängigen Variablen auch die Höhe der Beiträge erklären können. ${ }^{\circledR}$ Aus Platzgründen werden nun alle vier Modelle insgesamt betrachtet, um die Erklärungskraft der LZH zu eruieren. In vergleichbaren Untersuchungen übliche Variablen zum Informationsstand und kognitiven Fä-

(5) Bei der logistischen Regression werden die Koeffizienten ausgewiesen, da sie analog zu den Koeffizienten der linearen Regression in den Modellen 3 und 4 interpretierbar sind. Ein negatives Vorzeichen entspricht einem gegensätzlichen Zusammenhang.

(6) Die starke Streuung sowohl der Altersvorsorgebeiträge aber auch einiger unabhängiger Variablen (Einkommen, Vermögen, Ersparnisse) machten es notwendig, die jeweiligen Werte zu logarithmieren - andererseits wären ineffiziente Schätzer die Folge. Der Nachteil dieser Logarithmierung ist die erschwerte Interpretation der Effektgrößen. 


\section{Neigung zur privaten Altersvorsorge und Beitragshöhen}

\begin{tabular}{|c|c|c|c|c|c|c|c|c|}
\hline & \multicolumn{2}{|c|}{ (1) Riester-Rente (nein/ja) } & \multicolumn{2}{|c|}{ (2) pr. Vorsorge (nein/ja) } & \multicolumn{2}{|c|}{ (3) Riesterbeiträge } & \multicolumn{2}{|c|}{ (4) pr. Vorsorgebeiträge } \\
\hline & Koef. & SE & Koef. & SE & Koef. & SE & Koef. & SE \\
\hline Einkommen & -0.010 & $(0.15)$ & 0.167 & $(0.15)$ & $0.803^{* *}$ & $(0.12)$ & $0.945^{* *}$ & $(0.11)$ \\
\hline Einkommen ${ }^{2}$ & $-0.523 * *$ & $(0.14)$ & $-0.211 *$ & $(0.11)$ & 0.018 & $(0.14)$ & $0.217^{*}$ & $(0.10)$ \\
\hline Zukunft: mehr Einkommen & $0.045^{*}$ & $(0.02)$ & $0.085^{* *}$ & $(0.02)$ & -0.018 & $(0.02)$ & 0.008 & $(0.01)$ \\
\hline Einkommen früher schlechter & 0.000 & $(0.06)$ & 0.000 & $(0.05)$ & -0.001 & $(0.06)$ & $0.076^{+}$ & $(0.04)$ \\
\hline Alter & $-0.080 * *$ & $(0.01)$ & $-0.069 * *$ & $(0.01)$ & 0.003 & $(0.01)$ & -0.001 & $(0.01)$ \\
\hline Alter ${ }^{2}$ & $-0.003^{* *}$ & $(0.00)$ & $-0.003^{* *}$ & $(0.00)$ & & & & \\
\hline Myopie & -0.029 & $(0.03)$ & -0.017 & $(0.03)$ & 0.004 & $(0.03)$ & 0.012 & $(0.02)$ \\
\hline subjektive Lebenserwartung & 0.008 & $(0.01)$ & 0.007 & $(0.01)$ & -0.008 & $(0.01)$ & $-0.010^{+}$ & $(0.01)$ \\
\hline Geringe GRV Rente (nein/ja) & 0.166 & $(0.17)$ & $0.327^{+}$ & $(0.17)$ & -0.207 & $(0.16)$ & -0.046 & $(0.13)$ \\
\hline Hohe GRV Rente (nein/ja) & 0.134 & $(0.16)$ & $0.310^{+}$ & $(0.16)$ & 0.012 & $(0.16)$ & 0.072 & $(0.11)$ \\
\hline Dum. andere AV (nein/ja) & $0.527 * *$ & $(0.15)$ & & & -0.187 & $(0.14)$ & & \\
\hline betriebl. AV (nein/ja) & & & 0.136 & $(0.17)$ & & & -0.086 & $(0.12)$ \\
\hline Geldvermögen & $0.094^{* *}$ & $(0.02)$ & $0.130 * *$ & $(0.02)$ & 0.008 & $(0.02)$ & $0.028^{+}$ & $(0.02)$ \\
\hline Sparbeträge & 0.032 & $(0.02)$ & $0.046^{*}$ & $(0.02)$ & $0.038^{+}$ & $(0.02)$ & $0.034^{*}$ & $(0.02)$ \\
\hline Haushaltsgröße & $0.122^{+}$ & $(0.07)$ & 0.077 & $(0.08)$ & $-0.206^{*}$ & $(0.09)$ & $-0.122 *$ & $(0.06)$ \\
\hline Kinderanzahl & $0.209 * *$ & $(0.08)$ & 0.077 & $(0.09)$ & 0.009 & $(0.11)$ & -0.030 & $(0.07)$ \\
\hline Ostdeutschland (nein/ja) & $0.418 * *$ & $(0.15)$ & $0.315^{*}$ & $(0.15)$ & $-0.281 *$ & $(0.14)$ & $-0.176^{+}$ & $(0.10)$ \\
\hline Konstante & $-2.424 * *$ & $(0.36)$ & $-1.673^{* *}$ & $(0.34)$ & $4.468 * *$ & $(0.36)$ & $4.151^{* *}$ & $(0.24)$ \\
\hline $\mathrm{N}$ & 1.548 & & 1.548 & & 303 & & 529 & \\
\hline mittl. $\mathrm{R}^{2} / \mathrm{McF} . \mathrm{R}^{2}$ & 0.17 & & 0.20 & & 0.23 & & 0.29 & \\
\hline
\end{tabular}

Anmerkungen: Daten gewichtet und mehrfach imputiert; Variablenhinweise: Nettohaushaltseinkommen, Geldvermögen, Sparbeträge, Riesterbeiträge und private Vorsorgebeiträge logarithmiert, GRV Rentenerwartungen am Median dichotomisiert (Referenz: keine Angaben), private Vorsorge (Riester-Rente, private Rentenve sicherung, teilw. Lebensversicherung), Myopie wenn unmittelbare Resultate wichtiger als zukünftige, Lebenserwartung = erwartetes Alter; Einkommen, Alter, subjektive Lebenserwartung, Haushaltsgröße wurden zum Median hin zentriert; Signifikanz: ${ }^{*} p<0.1,{ }^{*} p<0.05,{ }^{* *} p<0.01$.

Quelle: Datenbasis SAVE 2008 (ohne Rentner und Selbstständige); Berechnungen des Autors.

higkeiten werden explizit nicht in das Modell aufgenommen, um möglichst nah an der Grundvariante der LZH zu bleiben.

Die Auswertung (Tabelle 2) zeigt: Das Einkommen eignet sich wie erwartet sehr gut zur Vorhersage von Alterssparentscheidungen. Während die Entscheidung für oder gegen Alterssparen entsprechend den theoretischen Überlegungen eine hügelförmige Verlaufsform annimmt (Abbildung 1), zeigt sich für die Höhe der Sparbeträge ein überproportional ansteigender Beitrag. ${ }^{\circ}$ Hier bestätigt sich ein aus der Forschung schon bekanntes Ergebnis, dass einkommensstarke Haushalte nicht nur absolut, sondern auch relativ mehr Geld sparen (Browning/Lusardi 1996, S. 1815). Es ist allerdings fraglich, ob diese Ergebnisse als Bestätigung der LZH interpretiert werden können. Der überproportionale Anstieg der Altersvorsorgeaufwendungen mit dem Einkommen bleibt auch unter Kontrolle der Proxy Variablen für das permanente Einkommen bestehen. Ergo: Die hohe Sparneigung der Hocheinkommensbezieher ergibt sich im Gegensatz zu den Überlegungen der LZH auch unter Berücksichtigung erwarteter Einkommenszuwächse sowie der Einkommensentwicklung in der Vergangenheit. In den logistischen Regressionsmodellen zeigt sich bei glei- chem Einkommen sogar eine höhere Sparneigung für Haushalte mit einer erwarteten Einkommenssteigerung (also einem höheren Permanenteinkommen) - was den Grundaussagen der LZH gänzlich widerspricht.

Zwischen dem Alter und dem Vorsorgeverhalten gibt es den aus den theoretischen Überlegungen abgeleiteten hügelförmigen Zusammenhang. ${ }^{8}$ Dass es sich hierbei aber tatsächlich um Effekte handelt, die durch Abschlusskosten erzeugt werden, oder ob sich hier stattdessen schlicht eine altersbedingte Aufmerksamkeit für Fragen der Altersvor-

(7) Ein hügelförmiger Verlauf lässt sich durch die zusätzliche Aufnahme eines quadratischen Terms modellieren. Ein negativer (positiver) quadratischer Term zeigt dabei eine nach unten (oben) geöffnete Parabel an.

(8) In den Modellen 3 und 4 wurde für die Vorhersage der Alterseffekte kein hügelförmiger Verlauf modelliert, da dort nur Personen befragt werden, die bereits Altersvorsorgeverträge haben. Der Einfluss hoher Abschlusskosten spielt dann keine Rolle mehr. Zusätzlich getestete Modelle zeigten auch keine kurvilinearen Effekte. 
sorge niederschlägt, bleibt offen. Für Letzteres spricht z. B., dass es innerhalb der Gruppe der „Vorsorgenden“ keine Alterseffekte mehr gibt. Eventuell sind es daher weniger Optimierungskalküle im Lebenszyklus, sondern vielmehr mit Altersstufen verbundene, sozial geformte Vorstellungen, wann Altersvorsorge betrieben werden sollte (Starr 2009). Diese Vermutung ist auch deshalb plausibel, weil die geringe Vorsorgeneigung in jungen Jahren selbst unter Kontrolle des individuellen Zeithorizonts bestehen bleibt - also nicht nur ein Ausdruck kurzsichtig handelnder Akteure sein kann. ${ }^{\ominus}$

Anders als die klassische LZH nahelegen würde, optieren Personen mit einer subjektiv höheren Lebenserwartung (unter Kontrolle anderer Variablen) weder besonders oft für Leibrenten, noch zeigen sie insgesamt eine erhöhte Alterssparaktivität. Die Grundaussage der LZH, einer Kalkulation der Ersparnisse im Hinblick auf den gesamten Lebenslauf, erscheint damit einmal mehr fraglich. Statt eine zentrale Zielvariable für Alterssparentscheidungen zu sein, spielt die Lebenserwartung keine nachweisbare Rolle bei der Vorsorge.

Wie sieht es nun mit dem Verhältnis zwischen dem privaten Alterssparen und alternativen Einkommensquellen im Alter aus? Da der Vorsorgebedarf aus verschiedenen Quellen bedient werden kann, sollten jene Haushalte, die auf zusätzliche finanzielle Ressourcen zurückgreifen können, bei gleichem Einkommen weniger private Altersvorsorge betreiben als Haushalte mit geringeren finanziellen Ressourcen. Im Gegensatz zu den Überlegungen aus der LZH deuten die Koeffizienten auf eine positive Beziehung hin. Das heißt: Haushalte, die bereits vergleichbar gut abgesichert sind, zeigen ein besonders ausgeprägtes Vorsorgeverhalten. Zum einen führt der Besitz anderer Altersvorsorgeprodukte tendenziell zu einer erhöhten Chance, private Altersvorsorgeprodukte abzuschließen - die Chance, eine Riester-Rente zu besitzen, erhöht sich beispielsweise bei gleichzeitigem Besitz anderer Vorsorgeprodukte um $69 \%$. Zum anderen geht die Erwartung niedriger gesetzlicher Renten nicht mit erhöhten Alterssparaktivitäten einher. ${ }^{\circledR}$ Das Geldvermögen aus dem Vorjahr und Altersvorsorgebereitschaft stehen sogar in einer positiven Beziehung zueinander. Haushalte, die im Vorjahr ein höheres Vermögen hatten, sparen bei gleichem Einkommen im Folgejahr nicht nur häufiger, sondern auch höhere Beträge für die Altersvorsorge als Haushalte mit geringem Vermögen. Ähnlich, aber nicht in allen Modellen signifikant, ist der Zusammenhang zwischen allgemeinem Sparverhalten und den Altersvorsorgeaufwendungen. ${ }^{\text {[D }}$ Sowohl für die staatlich geförderte Altersvorsorge als auch bei der allgemeinen Betrachtung der Altersvorsorge ergibt sich damit das Gesamtbild, dass jene Haushalte, die schon günstig mit finanziellen Mitteln ausgestattet sind, häufiger und mehr für das Alter sparen. Die sozialpolitisch motivierten finanziellen Anreize der Riester-Rente scheinen deshalb nur bedingt geeignet zu sein, bisher vorsorgeferne Bevölkerungsgruppen anzusprechen.
Die Haushaltszusammensetzung spielt auf zwei Ebenen eine zu erwartende Rolle: Zum einen zeigt sich, dass kinderreiche Haushalte eher „riestern“, d. h. die sozialpolitischen Anreize der Kinderzulagen wirken positiv auf den Abschluss von Riesterverträgen. Zum anderen bedeuten mehr Kinder eine höhere finanzielle Belastung - die entsprechenden Effekte zeigen sich bei der Höhe der Altersvorsorgebeiträge. Der positive Förderanreiz für Kinder wirkt sich nicht mehr positiv auf die Höhe der Altersvorsorgebeiträge aus, im Gegenteil: Bei steigender Haushaltsgröße wird weniger gespart (Tabelle 2, Modelle 3 und 4). Die Kontrollvariable für die neuen Bundesländer soll der Vollständigkeit halber nur noch erwähnt werden: Bewohner der neuen Länder haben zwar eine höhere Chance, private Vorsorge zu betreiben, gleichzeitig sind die Vorsorgebeiträge aber signifikant geringer.

\section{Diskussion}

Werden die Ergebnisse aus dem Blickwinkel der sozialpolitischen Förderung rekapituliert, so wird deutlich, dass diese Anreize nur bedingt wirken: So sind erstens jene Gruppen, die bisher kaum gespart haben, auch bei der staatlich geförderten Altersvorsorge weniger aktiv (junge Menschen, Haushalte mit niedrigen Einkommen). Zweitens werden zwar über die Kinderzulagen bisher der Altersvorsorge eher ferne Gruppen erreicht, die Höhe der Ersparnisse kann dadurch aber nicht stimuliert werden. Vom Abschluss eines Riester-Vertrags sollte daher

(9 In zusätzlichen Modellen wurde geprüft, ob es einen Interaktionseffekt zwischen dem Alter und dem individuellen Zeithorizont gibt. Dies entspräche den Überlegungen von Carroll und Samwick (1997), dass Altersvorsorge bei Haushalten mit kurzem Zeithorizont erst viel später in den Blick gerät. Diese Vermutung lässt sich allerdings nicht belegen.

(10 Die Haushalte wurden befragt, wie hoch sie ihre Lohnersatzleistungen aus der Rentenversicherung einschätzen. Die Angaben mussten aufgrund der hohen Anzahl fehlender Werte in mehrere Dummyvariablen zerlegt werden (keine Angabe/hohes Rentenniveau/niedriges Rentenniveau). Ein niedriges erwartetes Rentenniveau besteht dann, wenn die Lohnersatzrate unter $60 \%$ des letzten Nettolohns geschätzt wird.

(11) Eigentlich wurde der Sparindikator derart erhoben, dass darin auch Altersvorsorgebeiträge enthalten sind. Aufgrund des Antwortverhaltens in SAVE und Hinweisen aus anderen Studien (Essig 2005; Wilke 2010, S. 74) ist allerdings davon auszugehen, dass die Altersvorsorgeersparnisse in der Regel von den Befragten nicht berücksichtigt wurden. Um dennoch eine mögliche Doppelerfassung weitestgehend auszuschließen, wurden von den genannten Sparbeträgen die Altersvorsorgebeiträge abgezogen, wenn diese $60 \%$ der Gesamtersparnis nicht überschreiten. 
noch nicht auf die tatsächlichen Altersvorsorgeersparnisse geschlossen werden. Die bisher vorherrschenden Debatten um adäquate Vorsorge (z. B. Lamping/Tepe 2009; Börsch-Supan et al. 2008) sollten deshalb neben der Kontrahierung zunehmend auch die Höhe des Vorsorgesparens in den Blick nehmen. Aus sozialpolitischer Perspektive, so macht die hier vorgestellte Analyse deutlich, kann die empirisch beobachtbare Verbreitung der Riester-Rente allein keinesfalls mit dem Erreichen der sozialpolitischen Zielsetzungen gleichgesetzt werden. Der Erfolg der Riester-Rente ergibt sich nur dann, wenn auch hinreichend gespart wird - und hier zeigen die sozialpolitischen Anreize deutlich weniger Wirkung.

Im Gesamtbild aller vier Modelle (Tabelle 2) ergibt sich der Eindruck einer Kluft im Sparverhalten. Auf der einen Seite sind Haushalte, die aktiv Altersvorsorge betreiben. Gleichzeitig sind diese aktiven Haushalte durch ihr Einkommen sowie verschiedene Vorsorgeprogramme und Vermögenswerte sowieso schon überdurchschnittlich abgesichert. Auf der anderen Seite sind dagegen jene Haushalte mit geringen Vermögenswerten und Anspruchsrechten, die es aus hier nicht untersuchten Gründen scheinbar nicht schaffen, diese Lücke mit privater Altersvorsorge selbstständig zu schließen.

Damit wird deutlich, dass sich das in der angelsächsischen Forschung vorherrschende, aber auch im deutschen Diskurs verwendete Modell eines rational über den Lebenszyklus handelnden Nutzers zur Erklärung des Vorsorgeverhaltens kaum eignet. Die Lebenszyklusthese zeigt sich als allgemeines Erklärungsmodell wenig brauchbar und das, obwohl sie von der theoretischen Ausformulierung her geradezu auf die Erklärung von Altersvorsorgeentscheidungen zugeschnitten zu sein scheint. Weder konnte für diese repräsentative Stichprobe eine auf das gesamte Leben hin ausgerichtete Kalkulation nachgewiesen werden, noch konnten überzeugende Belege für ein am Permanenteinkommen ausgerichtetes Sparverhalten erbracht werden. Und schließlich lässt sich keine auf das gesamte Vermögen ausgerichtete Sparstrategie nachweisen.

Der enge, ausschließlich am rationalen Nutzer angelegte Diskurs scheint aus dem Blickwinkel dieses Aufsatzes als deutlich verkürzt. Es stellt sich daher unmittelbar die Frage, ob neben dem rationalen Nutzer auch andere Nutzerrollen in Erscheinung treten, die das Selbstverständnis in Bezug auf ihre Altersvorsorge besser abbilden. Vereinzelt wurden bereits Anstrengungen unternommen, den rationalen Nutzer umfassender darzustellen und ihm psychologische Einschränkungen und Informationsdefizite zuzugestehen. Gleichzeitig wurde aber die Rolle des rationalen Nutzers als leitend beibehalten. Es erscheint daher vielversprechend, private Altersvorsorge auch aus einer spezifisch soziologischen Perspektive heraus zu analysieren. Neben der sozialen und institutionellen Einbettung der Akteure könnten auch Sozialisationserfahrungen und bestimmte Wertauffassungen oder Einstellungsmuster (z. B. Lamping/Tepe 2009) wichtige Einblicke in das Selbstverständnis der Nutzer beim Umgang mit privater Altersvorsorge liefern und einen Beitrag dazu leisten, die beobachteten Heterogenitäten im Sparverhalten besser zu verstehen. ${ }^{\text {(1) }}$

Die Befunde provozieren dazu, mögliche gesellschaftspolitische Konsequenzen zu eruieren. Die politische Ausgestaltung des Altersvorsorgemarktes, bei der ausschließlich auf rational planende Nutzer gesetzt wurde,

(12) In unserem aktuellen Forschungsprojekt "Orientierungssuche bei der privaten Altersvorsorge" (Bode/Wilke) an der Universität Kassel versuchen wir beispielsweise, die Rolle der Einbettung in soziale Netzwerke auf private Altersvorsorge zu analysieren. lässt bereits Mängel für das Gesamtversorgungsniveau der Bevölkerung erahnen, die in den vorstehenden Ausführungen bestätigt wurden. Zum Ersten ist die Verstärkung sozialer Ungleichheiten schon jetzt absehbar. In Niedrigeinkommenshaushalten wird es eine beträchtliche Anzahl von Haushalten nicht schaffen, adäquat für das Alter vorzusorgen. An dieser Stelle ist ein möglichst frühes politisches Eingreifen notwendig, um diese Fehlentwicklungen zu korrigieren. Zum Zweiten ist davon auszugehen, dass die beobachtete Heterogenität im Sparverhalten innerhalb der einzelnen sozio-ökonomischen Statusgruppen auch längerfristig bestehen bleibt. Damit werden sehr wahrscheinlich auch Haushalte, die von ihrer ökonomischen Ausgangslage eigentlich gut abgesichert wären, aufgrund von (in der ex-post Betrachtung) suboptimalen Anlageentscheidungen mit dem Thema Altersarmut konfrontiert. Mit finanziellen Anreizen allein, die ausschließlich auf rationale Nutzer rekurrieren, ist ein solches Problem allerdings nur schwer zu lösen.

\section{LITERATUR}

Binswanger, J./Carman, K. G. (2009): How Real People Make Long-Term Decisions, in: Center Discussion Paper (73), Tilburg

Blank, F. (2011): Wohlfahrtsmärkte in Deutschland, in: WSI-Mitteilungen 64 (1), S. 11-18, http://www.boeckler.de/wsimit_2011_01_Blank.pdf

Blossfeld, H. (1989): Kohortendifferenzierung und Karriereprozess,

Frankfurt a. M.

Bode, I. (2007): Die institutionelle Dynamik im „welfare mix" der Alterssicherung, Wuppertal

Börsch-Supan, A./Coppola, M./Essig, L./Eymann, A./Schunk, D. (2008): The German SAVE study, MEA Studie (6), Mannheim

Börsch-Supan, A./Reil-Held, A./Schunk, D. (2006): Das Sparverhalten Deutscher Haushalte, MEA Discussion Papers (114), Mannheim

Browning, M./Lusardi, A. (1996): Household Saving: MicroTheories and Micro Facts, in: Journal of Economic Literature 34 (4), S. 1797-1855

Carroll, C. D./Samwick, A. A. (1997): The Nature of Precautionary Wealth, in: Journal of Monetary Economics 40 (1), S. 41-71

Corneo, C./Keese M./Schröder C. (2009): The Riester Scheme and private savings, in: Schmollers Jahrbuch 129 (2), S. 321-332

Duš, I./Maurer, R. (2007): Finanzierung des Ruhestands, Köln

Dynan, K. E./Skinner, J./Zeldes, S. P. (2004): Do the Rich Save More? in: Journal of Political Economy 112 (2), S. 397-444

Essig, L. (2005): Measures for Savings and Saving Rates in the German SAVE Data Set, Mea Discussion Papers 068-05, Mannheim

Feldstein, M. (1974): Social Security, Induced Retirement, and Aggregate Capital Accumulation, in: Journal of Political Economy 82 (5), S. 905-926 Frederick, S./Loewenstein, G./O'Donoghue, T. (2002): Time Discounting and Time Preference, in: Journal of economic Literature 40 (2), S. 351-401 Hinrichs, K. (2004): Alterssicherungspolitik in Deutschland: Zwischen Kontinuität und Paradigmenwechsel, in: Beyer, J./Stykow, P. (Hrsg.): Gesellschaft mit beschränkter Hoffnung, Wiesbaden, S. 266-286

Hockerts, H. G. (1985): Sicherung im Alter, in: Conze, W./Lepsius, R. M. (Hrsg.): Sozialgeschichte der Bundesrepublik Deutschand, Stuttgart, S. 296-323 Hühne, D./Balodis, H. (2010): Privatrenten und Lebensversicherungen, Düsseldorf

Kohlert, D./Oehler, A. (2009): Scheitern Finanzdienstleistungen am Verbraucher?, in: Vierteljahrshefte zur Wirtschaftsforschung 78 (3), S. $81-95$ Lamping, W./Tepe, M. (2009): Vom Können und Wollen der privaten Altersvorsorge, in: Zeitschrift für Sozialreform 55 (4), S. 409-430 
Lang, O. (1998): Steueranreize und Geldanlage im Lebenszyklus, Baden-Baden Legros, L. (2006): Life-Cycle Options and Preferences, in: Clark, G. L./Munnell, A. H./Orszag, M. J. (Hrsg.): Oxford handbook of pensions and retirement income, Oxford, S. 183-201

Leinert, J. (2003): Altersvorsorge 2003: Wer hat sie, wer will sie? Bertelsmann Stiftung, Vorsorgestudien (18), Gütersloh

Modigliani, F./Brumberg, R. (1954): Utility Analysis and the Consumption Function, in: Kurihara, K. K. (Hrsg.): Post-Keynesian Economics, New Brunswick, S. 388-436

Nüchter, O./Bieräugel, R./Schipperges, F./Glatzer, W./Schmid, W. (2008): Einstellungen zum Sozialstaat II, Opladen

Oehler, A. (2009): Alles „Riester" ? Die Umsetzung der Förderidee in der Praxis, Bamberg

Oehler, A./Werner, C. (2008): Saving for Retirement - A Case for Financial Education in Germany and UK?, in: Journal of Consumer Policy 31 (3), S. 253-283 Shefrin, H. M./Thaler, R. H. (1988): The Behavioral Life-Cycle Hypothesis, in: Economic Inquiry 26 (4), S. 609-643

Smyth, D. J. (1993): Toward a Theory of Saving, in: Gapinski, J. H. (Hrsg.): The Economics of Saving, Boston, S. 47-93
Starr, M. A. (2009): Lifestyle conformity and lifecycle saving, in: Cambridge Journal of Economics 33 (1), S. 25-49

Wilke, F. (2010): Sparen aus Ungewissheit, Kassel

\section{AUTOR}

FELIX WILKE, Dipl. Soz. MA (History), ist wissenschaftlicher Mitarbeiter an der Universität Kassel. Arbeitsschwerpunkte: Sozialpolitik, Soziologie des Sparens und der Vorsorge, Europasoziologie.

wilke@uni-kassel.de 